\title{
The Effects of Salts and Osmoprotectants on Enzyme Activities of Fructose-1,6-biphosphate Aldolases in a Halotolerant Cyanobacterium, Halothece sp. PCC 7418
}

\author{
Siripat Ngoennet ${ }^{1}$, Masaki Honda ${ }^{2}{ }^{\mathbb{D}}$, Tanutcha Patipong ${ }^{1}$, Takashi Hibino ${ }^{2,3}$, \\ Rungaroon Waditee-Sirisattha ${ }^{1, *(\mathbb{D})}$ and Hakuto Kageyama $2,3, *$ (D) \\ 1 Department of Microbiology, Faculty of Science, Chulalongkorn University, Payathai Road, Patumwan, \\ Bangkok 10330, Thailand; siripat.ngoennet@gmail.com (S.N.); tanutchapatipong@gmail.com (T.P.) \\ 2 Department of Chemistry, Faculty of Science and Technology, Meijo University, 1-501 Shiogamaguchi, \\ Tenpaku-ku, Nagoya, Aichi 468-8502, Japan; honda@meijo-u.ac.jp (M.H.); hibino@meijo-u.ac.jp (T.H.) \\ 3 Graduate School of Environmental and Human Sciences, Meijo University, 1-501 Shiogamaguchi, \\ Tenpaku-ku, Nagoya, Aichi 468-8502, Japan \\ * Correspondence: Rungaroon.W@chula.ac.th (R.W.-S.); kageyama@meijo-u.ac.jp (H.K.); \\ Tel.: +66-2-2185091 (R.W.-S.); +81-52-838-2609 (H.K.)
}

Received: 18 February 2020; Accepted: 6 March 2020; Published: 9 March 2020

\begin{abstract}
The halotolerant cyanobacterium, Halothece sp. PCC 7418, possesses two classes of fructose-1,6-bisphosphate aldolase (FBA): $\mathrm{H} 2846$ and H2847. Though class I (CI)-FBA H2846 is thought to be associated with salt tolerance, the regulatory mechanisms, molecular characteristics, and expression profiles between $\mathrm{H} 2846$ and class II (CII)-FBA H2847 have scarcely been investigated. Here, we show that the accumulation of the $\mathrm{H} 2846$ protein is highly responsive to both up- and down-shock with $\mathrm{NaCl}$, whereas $\mathrm{H} 2847$ is constitutively expressed. The activity of CI- and CII-FBA in cyanobacterial extracts is correlated with the accumulation patterns of $\mathrm{H} 2846$ and $\mathrm{H} 2847$, respectively. In addition, it was found that these activities were inhibited by $\mathrm{NaCl}$ and $\mathrm{KCl}$, with CII-FBA activity strikingly inhibited. It was also found that the CI-FBA activity of recombinant $\mathrm{H} 2846$ was hindered by salts and that this hindrance could be moderated by the addition of glycine betaine (GB), whereas no moderation occurred with other potential osmoprotectant molecules (proline, sucrose, and glycerol). In addition, a phylogenetic analysis showed that CI-FBAs with higher similarities to $\mathrm{H} 2846$ tended to be distributed among potential GB-synthesizing cyanobacteria. Taken together, our results provide insights into the independent evolution of the CI- and CII-FBA gene families, which show distinct expression profiles and functions following salt stress.
\end{abstract}

Keywords: fructose-1,6-bisphosphate aldolase; salt stress; halotolerant cyanobacteria; glycine betaine

\section{Introduction}

Fructose-1,6-bisphosphate aldolase (FBA), an enzyme, which is indispensable for glycolysis, gluconeogenesis, and the Calvin cycle, catalyzes the reversible aldol cleavage of fructose 1,6-bisphosphate (FBP) into glyceraldehyde 3-phosphate (G3P) and dihydroxyacetone phosphate (DHAP) (Figure 1) [1]. FBAs are classified into two distinct classes, according to their catalytic mechanism and independent evolutionary occurrence [2]. Class I (CI)-FBAs catalyze the reaction via the formation of a Schiff base, which attacks the $\mathrm{C} 1$ carbonyl group of G3P between the $\mathrm{C} 2$ carbonyl group of DHAP and the N6 amino group of an essential lysine residue in the active site. Class II (CII)-FBAs require divalent metal ions for a similar catalytic mechanism. It has been reported that CI-FBAs are commonly present in eukaryotic organisms, such as animals, plants, and green algae, 
but occur rarely in bacteria, whereas CII-FBAs are generally present in bacteria, archaea, and lower eukaryotes, such as fungi and some green algae [3].

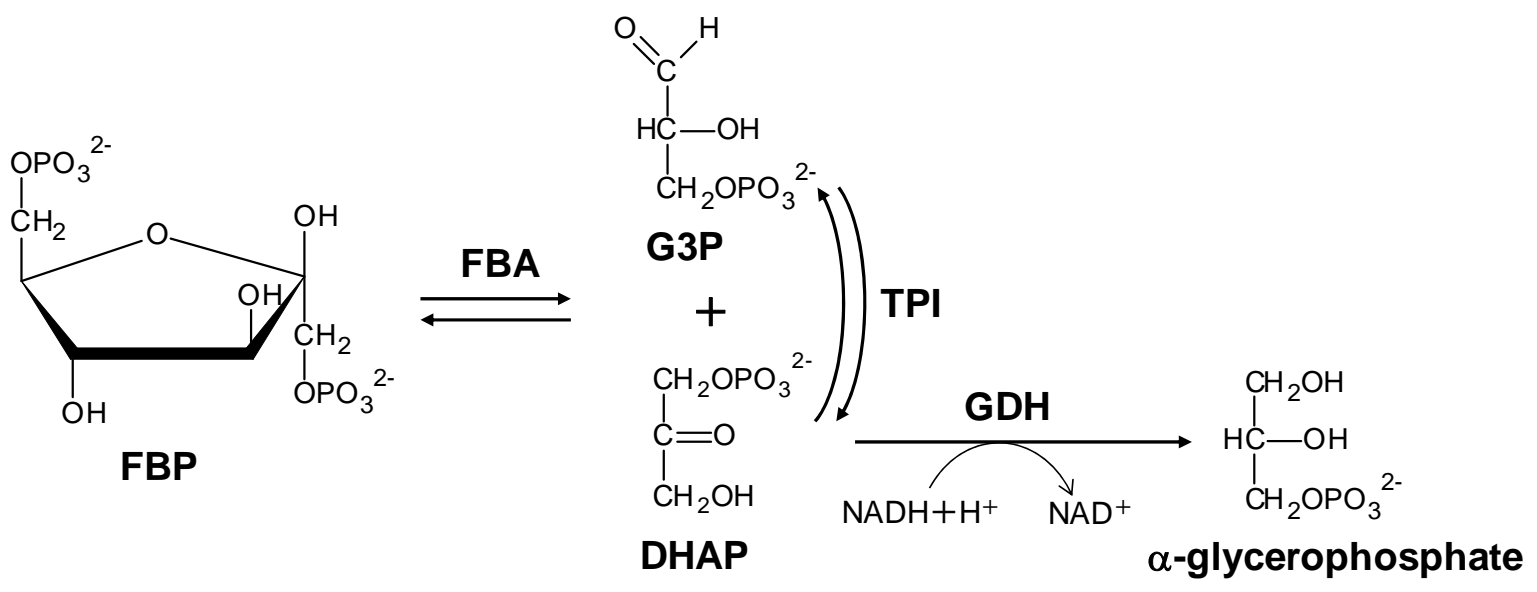

Figure 1. A coupled enzyme assay for the determination of FBP-cleavage activity. The enzymatic activity of FBA was measured by monitoring the decrease in the absorption of NADH at $340 \mathrm{~nm}$. FBP, fructose 1,6-bisphosphate; FBA, fructose-1,6-bisphosphate aldolase; G3P, glyceraldehyde-3-phosphate; DHAP, dihydroxyacetone phosphate; TPI, triose phosphate isomerase; and GDH, glycerol phosphate dehydrogenase.

Interestingly, based on genome sequence information, it has been shown that specific cyanobacterial species, including the cyanobacterium Halothece sp. PCC 7418 (Halothece 7418), possess CI-FBA in addition to CII-FBA [4]. Hereafter, we refer to Halothece 7418 CI-FBA and CII-FBA as H2846 and H2847, respectively, according to a previous report [4]. Halothece 7418, formerly identified as Aphanothece halophytica [5], is a halotolerant cyanobacterium, which was originally isolated from the Dead Sea, that can grow under high salinity concentrations of up to $3.0 \mathrm{M} \mathrm{NaCl}$ and an alkaline $\mathrm{pH}$ of 11 [6]. This strain is a remarkable example of cyanobacterial tolerance to high salt concentrations. To adjust their internal osmotic status under high salinity conditions, Halothece 7418 synthesizes and accumulates the osmoprotectant, glycine betaine (GB) via a three-step methylation reaction by two $N$-methyltransferases that utilizes glycine [7]. In addition to GB, it has been reported that another potential osmoprotectant, mycosporine-2-glycine (M2G), is also biosynthesized by Halothece $7418[8,9]$. However, the finding that the accumulated levels of GB were considerably higher than those of M2G [10] suggests that GB is the main osmoprotectant in Halothece 7418. Additionally, unique mechanisms for ion homeostasis in Halothece 7418 have been extensively investigated [11]. Very recently, we identified an H2846 protein as a salt-inducible CI-FBA in Halothece 7418 [4]. A phylogenetic analysis showed that H2846-like CI-FBAs are mostly present in cyanobacteria that inhabit hypersaline environments. The heterologous expression of $\mathrm{H} 2846$ but not $\mathrm{H} 2847$, a CII-FBA in Halothece 7418, could confer salinity tolerance to freshwater cyanobacterium. These results suggest a functional distinction between CI-FBA and CII-FBA in Halothece 7418, as well as the contribution of $\mathrm{H} 2846$ to salt tolerance mechanisms by the reinforcement of intracellular metabolic activities. However, the regulatory mechanisms, molecular characteristics, and expression patterns of these Halothece FBAs remain to be clarified. In the present study, we investigated the regulation of CI- and CII-FBA activity by confirming the levels of the accumulation of the $\mathrm{H} 2846$ and $\mathrm{H} 2847$ proteins in Halothece 7418 under salinity shock-treatment conditions. Our data revealed that only CI-FBA activity was highly responsive to both $\mathrm{NaCl}$ up- and down-shock treatments. The accumulation dynamics of CI-FBA (H2846) were highly induced upon salt stress. Furthermore, CI-FBA activity showed better resistance to salts than CII-FBA activity. Finally, we demonstrated that GB significantly alleviated the inhibitory effect of salt on the CI-FBA activity of the recombinant $\mathrm{H} 2846$ protein. These findings provide insights into the independent evolutionary history of the CI- and CII-FBA gene families, which exhibit distinct expression profiles and functions following salt stress. 


\section{Materials and Methods}

\subsection{Cyanobacterial Culture Conditions}

The cyanobacterium Halothece 7418 was photoautotrophically grown under continuous illumination of $70 \mu \mathrm{E} \mathrm{m}^{-2} \mathrm{~s}^{-1}$ at $30^{\circ} \mathrm{C}$ in a liquid BG11 plus Turk Island salt solution containing $0.5 \mathrm{M}$ or $2.5 \mathrm{M} \mathrm{NaCl}$. The medium was prepared according to a previously described recipe [12]. For the $\mathrm{NaCl}$ up-shock treatment, cells cultured in the media containing $0.5 \mathrm{M} \mathrm{NaCl}$ for at least 10 days were harvested by centrifugation and then resuspended in the media containing $2.5 \mathrm{M} \mathrm{NaCl}$. Conversely, cells cultured in the media containing $2.5 \mathrm{M} \mathrm{NaCl}$ were moved into the media containing $0.5 \mathrm{M} \mathrm{NaCl}$ for the $\mathrm{NaCl}$ down-shock treatment.

\subsection{Preparation of Rabbit Antiserum Directed Against H2847 Protein}

A white rabbit was immunized four times with a total of $1.0 \mathrm{mg}$ of synthesized peptide that corresponded to the region 289-306, REAAMKDPANFDPRHFLK, in the amino acid sequence of H2847 (Sigma-Aldrich Japan, Tokyo, Japan). The specificity of the antiserum for the H2847 protein was assessed by immunoblotting, which revealed that the antiserum specifically detected a band that was consistent with the predicted molecular mass (38.9 kDa) of H2847.

\subsection{Preparation of Soluble Protein Extracts of Halothece 7418 for Measurement of FBA Activity}

Halothece 7418 cells were collected from $50 \mathrm{~mL}$ of the culture during the log phase $\left(\mathrm{OD}_{730}=0.6 \sim 0.9\right)$ and then stored at $-80^{\circ} \mathrm{C}$ until use. Cells were suspended in $700 \mu \mathrm{L}$ of $50 \mathrm{mM}$ Tris- $\mathrm{HCl}(\mathrm{pH} 8.0)$ and sonicated on ice by using a VP-5s sonicator (TAITEC, Saitama, Japan) for a total of $40 \mathrm{~s}$ (repeated time-on/time-off of $10 \mathrm{~s}$ each time), with the output power set to 7 . The samples were then centrifuged at $22,000 \times \mathrm{g}$ for $10 \mathrm{~min}$ at $4{ }^{\circ} \mathrm{C}$. The supernatant solutions were used as total soluble protein extracts. Protein concentration was determined by using a TaKaRa Bradford Protein Assay Kit (Takara Bio Inc., Shiga, Japan).

\subsection{Preparation of Purified Recombinant H2846 Protein}

The recombinant H2846 protein was prepared as previously described [4]. Briefly, the H2846 protein was expressed in a soluble form in Escherichia coli BL21, which harbored the expression vector pColdI-H2846; the protein was then extracted and purified. The purification process consisted of two chromatographic steps: affinity purification that used an Ni-NTA-spin kit column (Qiagen, Hilden, Germany) and size-exclusion chromatographic purification that used a HiLoad 16/600 Superdex 200 pg column (GE Healthcare Life Sciences, Little Chalfont, United Kingdom).

\subsection{Measurement of FBA Activity}

The FBP-cleavage activity of FBA was measured by using a coupled enzyme assay. The reactions that were involved in the assay are illustrated in Figure 1.

To measure CI-FBA activity, an assay was performed according to a previously described method [4]. Briefly, the reaction mixture contained $50 \mathrm{mM}$ Tris- $\mathrm{HCl}(\mathrm{pH}$ 8.0), $0.2 \mathrm{mM} \mathrm{NADH}, 1$ unit of glycerol phosphate dehydrogenase and triose phosphate isomerase (GDH-TPI coupling enzyme; Sigma-Aldrich, St. Louis, MO, USA), $100 \mu \mathrm{M}$ FBP, and $5 \mathrm{mM}$ ethylenediaminetetraacetic acid (EDTA). EDTA was added to inhibit the CII-FBA activity. Following the addition of the Halothece 7418 soluble protein extract $(20 \mu \mathrm{g}$ of total protein) or the purified $\mathrm{H} 2846$ protein $(1 \mu \mathrm{g})$ to the reaction mixture, a decrease in absorbance at $340 \mathrm{~nm}$ was monitored by using a UV-1800 spectrophotometer (Shimadzu, Kyoto, Japan). The total volume of the reaction mixture was $500 \mu \mathrm{L}$. The reaction was performed at $30{ }^{\circ} \mathrm{C}$. 
To measure CII-FBA activity, a similar assay was carried out. However, the reaction mixture contained $2 \mathrm{mM} \mathrm{MnCl}_{2}$ to activate CII-FBA. Additionally, $10 \mathrm{mM}$ sodium borohydride was supplied, rather than EDTA, to inhibit CI-FBA activity.

To investigate the effect of salts $(\mathrm{NaCl}$ or $\mathrm{KCl})$ or osmoprotectants $(\mathrm{GB}$, proline, sucrose, or glycerol) on FBA activity, these compounds were dissolved in the reaction mixture at the indicated concentrations before the enzyme solution was added. Prior to conducting the analysis for the effect of salt, it was confirmed that $\mathrm{NaCl}$ and $\mathrm{KCl}$ did not inhibit the activity of the GDH-TPI coupling enzyme by using G3P as a substrate (data not shown).

One unit of FBA activity was defined as the amount of FBA that was required for the cleavage of $1 \mu \mathrm{mol}$ of FBP (equivalent to $2 \mu \mathrm{mol}$ of oxidized NADH) in $1 \mathrm{~min}$. The molar extinction coefficient for NADH $\left(6220 \mathrm{M}^{-1} \mathrm{~cm}^{-1}\right)$ was used for the calculation.

\subsection{Gel Filtration Chromatography of Halothece Extracts}

Halothece 7418 cells that were cultured under $2.5 \mathrm{M} \mathrm{NaCl}$ conditions were collected from $130 \mathrm{~mL}$ of the culture during the log phase $\left(\mathrm{OD}_{730}=0.6 \sim 0.9\right)$ and then stored at $-80{ }^{\circ} \mathrm{C}$ until use. Cells were suspended in $2.4 \mathrm{~mL}$ buffer A ( $50 \mathrm{mM}$ Tris- $\mathrm{HCl}$ ( $\mathrm{pH} 8.0), 150 \mathrm{mM} \mathrm{NaCl}, 5 \mathrm{mM} \mathrm{MgCl}_{2}$ ) and sonicated on ice by using a VP-5s sonicator (TAITEC) for a total of $40 \mathrm{~s}$ (repeated time-on/time-off of $10 \mathrm{~s}$ each time), with the output power set to 7 . The samples were then centrifuged at $22,000 \times \mathrm{g}$ for $15 \mathrm{~min}$ at $4{ }^{\circ} \mathrm{C}$. The supernatant solutions were passed through a membrane filter (pore size: $0.45 \mu \mathrm{m}$ ) and then subjected to size-exclusion separation by using a HiLoad 16/600 Superdex $200 \mathrm{pg}$ column. Buffer A was used as the eluent. Note that $\mathrm{MgCl}_{2}$ was used instead of $\mathrm{MnCl}_{2}$ in buffer $\mathrm{A}$ because manganese can be oxidized by Tris to form precipitate over a period of hours [13]. All protein chromatographic steps were performed by using the AKTA start system (GE Healthcare Life Sciences) at $4{ }^{\circ} \mathrm{C}$. During separation, buffer A was applied at a flow rate of $2.0 \mathrm{~mL} / \mathrm{min}$, and separated protein fractions were continuously collected every $2.0 \mathrm{~mL}$. The protein standards that were used for calibration were ferritin $(440 \mathrm{kDa})$, aldolase (158 kDa), conalbumin (75 kDa), ovalbumin (43 kDa), chymotrypsinogen A (25 kDa), and ribonuclease A ( $13.7 \mathrm{kDa})$; all were purchased from GE Healthcare Life Sciences. The proteins in $1.0 \mathrm{~mL}$ aliquots of the fractions were precipitated with trichloroacetic acid (TCA), and then the precipitates were dissolved in a $50 \mu \mathrm{L}$ SDS sample buffer and boiled for $5 \mathrm{~min}$.

\subsection{Immunoblotting Analysis}

For the immunoblotting analysis of the Halothece 7418 soluble protein extracts, $3.0 \mu \mathrm{g}$ of total protein samples were subjected to SDS-PAGE on $12 \%$ gels and blotted onto polyvinylidene difluoride (PVDF) membranes. For the immunoblotting analysis of gel filtration chromatographic fractions, $5 \mu \mathrm{L}$ of each sample was used. H2846 and H2847 were analyzed with specific antisera at a 1:1000 dilution and detected by a colorimetric method by using the alkaline phosphatase-conjugated secondary antibody. Anti-H2846 antiserum was prepared previously [4].

\subsection{Phylogenetic Analysis}

Cyanobacterial H2846-like proteins were extracted by using the National Center for Biotechnology Information (NCBI) protein BLAST algorithm, by searching with the amino acid sequence of H2846 as a protein query. The reliability of the phylogenetic tree (Figure 7) was assessed with a bootstrap analysis with 1000 replicates.

\section{Results and discussion}

\subsection{Protein Accumulation Dynamics of H2846 and H2847 Following Salinity Shock Treatment}

First, we investigated the accumulation pattern of CI-FBA H2846 under salt-shock conditions in Halothece 7418 cells. In this study, both $\mathrm{NaCl}$ up-shock $(0.5 \mathrm{M}$ to $2.5 \mathrm{M})$ and down-shock $(2.5 \mathrm{M}$ to $0.5 \mathrm{M}$ ) treatments were tested. As shown in Figure 2A, the immunoblotting analysis revealed that 
H2846 accumulation was significantly enhanced following an up-shock, as previously reported [4], and the level increased by approximately seven-times within $48 \mathrm{~h}$ following treatment. By contrast, the salt down-shock treatment caused a rapid decrease in H2846 accumulation. Thus, H2846 accumulation was highly responsive to the salinity shock treatment.
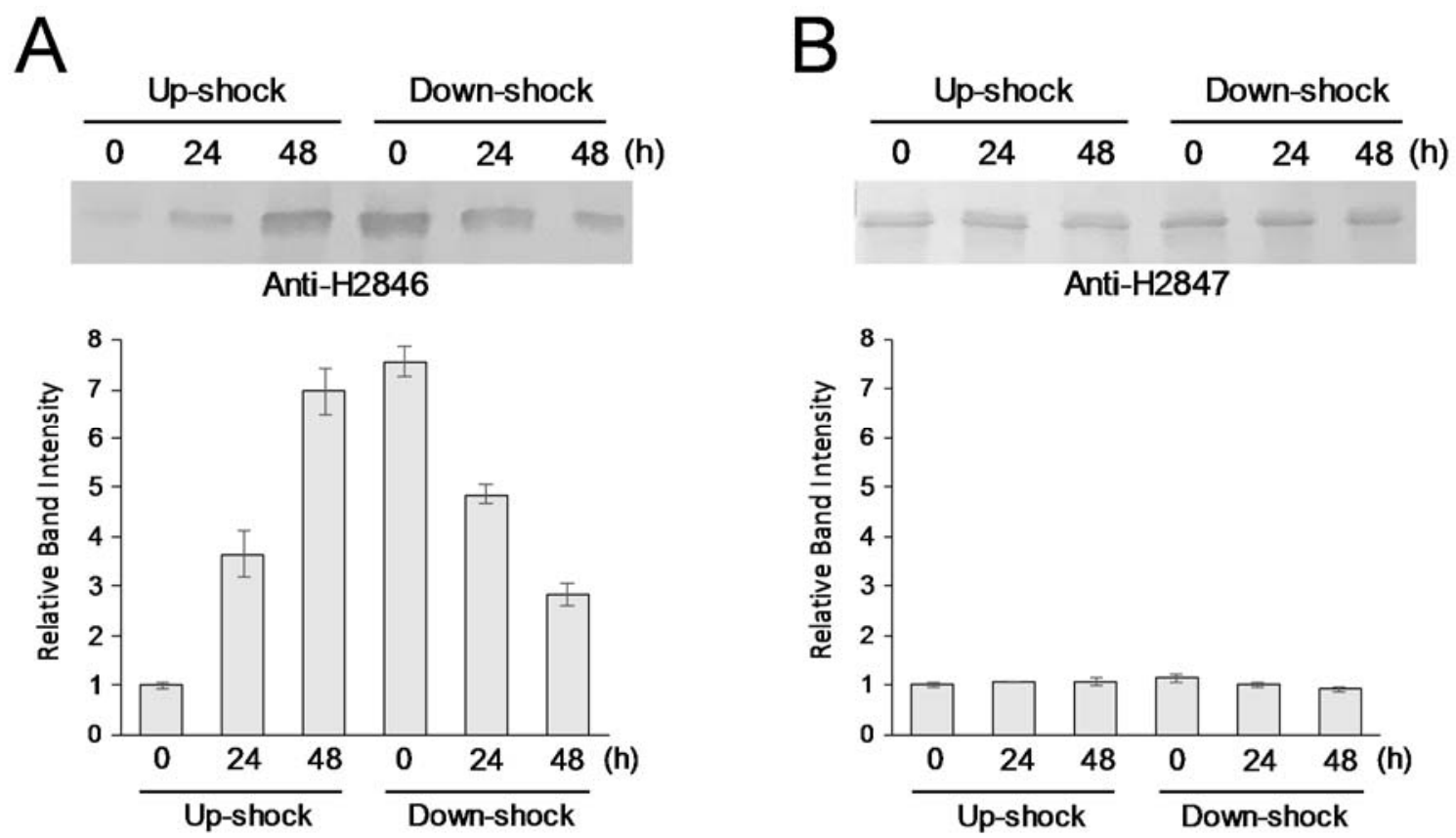

Figure 2. Accumulation dynamics of H2846 (A) and H2947 (B). Upper panels: Target FBAs were analyzed by immunoblotting by using corresponding antisera. Lower panels: densitometric data of the upper panels are shown. Each value represents the average of three independent analyses. Means \pm SEM are shown.

The accumulation of CII-FBA H2847 was examined in parallel. To do this, antiserum against the H2847 protein was newly prepared for the immunoblotting analysis (see Materials and Methods). As shown in Figure 2B, although our previous data demonstrated a slight upregulation of $H 2847$ gene expression following $\mathrm{NaCl}$ up-shock treatment [4], the level of accumulation of the $\mathrm{H} 2847$ protein was unchanged under both the up- and down-shock-treated conditions. A constant amount of H2847 might function as housekeeping FBA, which is involved in fundamental metabolism in Halothece 7418. The fact that H2847-type CII-FBA is highly conserved across a broad range of cyanobacterial species [4] supports this hypothesis.

In addition, we examined the complex formation of FBAs in Halothece cells that were cultured under salt stress $(2.5 \mathrm{M} \mathrm{NaCl})$ conditions. The gel filtration analysis followed by immunoblotting demonstrated that $\mathrm{H} 2846$ eluted as a single peak at a size larger than $158 \mathrm{kDa}$, as previously reported (Figure 3A) [4]. Through the use of HPLC-based size-exclusion chromatography, it has been suggested that $\mathrm{H} 2846$ forms a homohexamer by using a recombinant protein [4]. On the other hand, here, H2847 eluted as two peaks: one peak at a size larger than $158 \mathrm{kDa}$ and another peak at a size between 25 and $43 \mathrm{kDa}$ (Figure 3B). The predicted molecular mass of H2847 was $38.9 \mathrm{kDa}$, so it is likely that H2847 exists as both protein complexes and monomeric forms in almost the same ratio (Figure $3 \mathrm{~B}$ ). If the complex formed was a homo-oligomer, it should have been larger than a tetramer (155.6 kDa). This observation is interesting, because CII-FBAs of both bacterial and eukaryotic origin have been reported to commonly form homodimers [14]. 


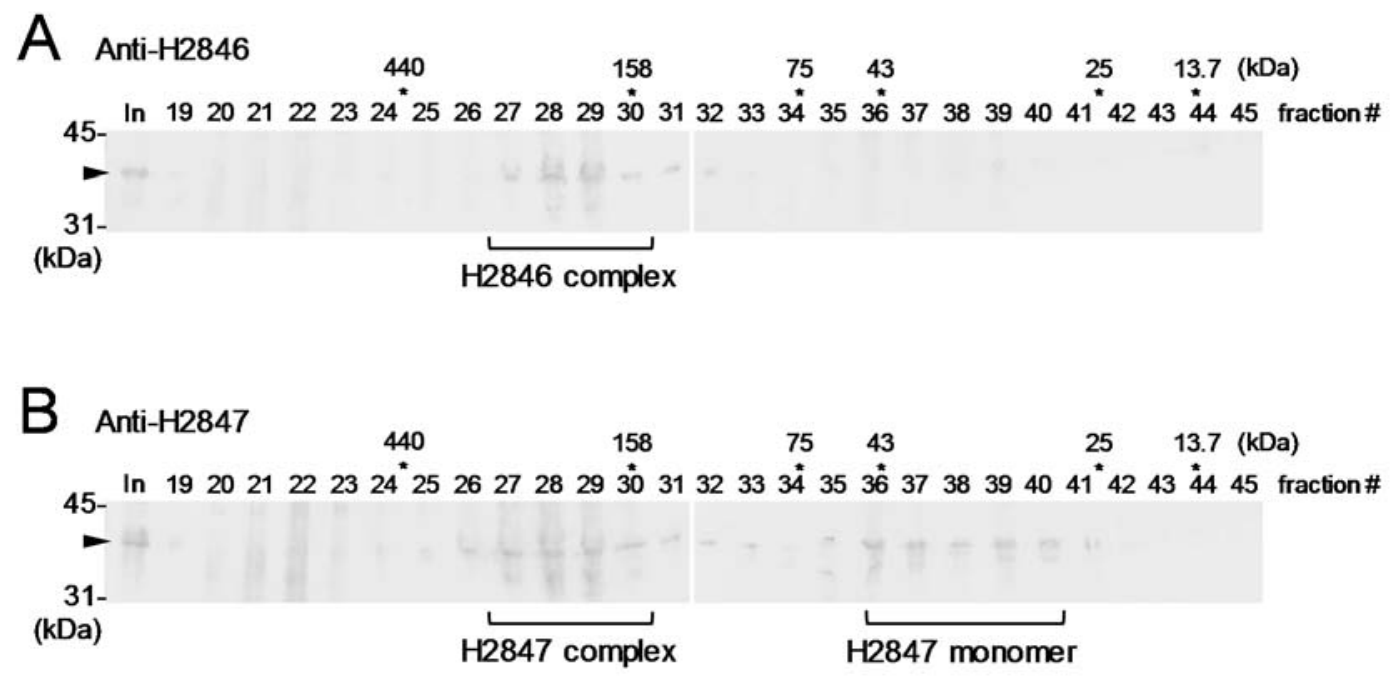

Figure 3. Complex formation of $\mathrm{H} 2846$ (A) and $\mathrm{H} 2847$ (B) in Halothece 7418 cells. The size-fractionated soluble protein extracts of Halothece 7418 by gel filtration analyses were subjected to immunoblotting by using corresponding antisera. The numbers upper the pictures indicate the number of fractions. The asterisks indicate the peaks of standard proteins and their native molecular size. 'In' indicates input, which was $3.0 \mathrm{mg}$ of the total protein sample of the Halothece soluble protein extract that was prepared from the cells that were sampled $48 \mathrm{~h}$ following $\mathrm{NaCl}$ up-shock treatment. The arrowheads indicate the bands of target FBAs. The peak areas corresponding to putative monomeric form and/or complexes are marked under the bands.

\subsection{FBA Activity under Salinity-Shocked Conditions in Halothece 7418}

Next, the FBA activity of Halothece 7418 extracts was examined. To measure the activity of CI-FBA and CII-FBA, EDTA, and sodium borohydride were added as respective inhibitors of the opposite activity (see Materials and Methods).

As shown in Figure 4A, CI-FBA activity was upregulated and downregulated by up- and down-shock with $\mathrm{NaCl}$, respectively. The patterns correlated well with the accumulation profile of the H2846 protein (Figure 2A).

To measure CII-FBA activity, divalent metal ion dependency was investigated first, because this class of FBAs requires divalent ions for their activity. For example, it was reported that cobalt (II) ions could have the best effect for activating CII-FBA, the sll0018 protein, in the cyanobacterium Synechocystis sp. PCC6803 [15]. To this end, eight divalent ions were tested in the presence of sodium borohydride, and it was found that the addition of manganese (II) ions resulted in the highest CII-FBA (H2847) activity in the Halothece 7418 extract (Table 1). The difference in divalent ion dependency between $\mathrm{H} 2847$ and sll0018 is an interesting subject that should be further clarified, since the amino acid sequences of these CII-FBAs were highly conserved ( $85 \%$ identity). Then, manganese (II) ions were added to the reaction mixtures for the CII-FBA assay by using the Halothece 7418 extracts. As a result, CII-FBA activity was not significantly changed by either the up- or down-shock treatments (Figure 4B). This result was in accordance with the accumulation pattern of the H2847 protein (Figure 2B). 

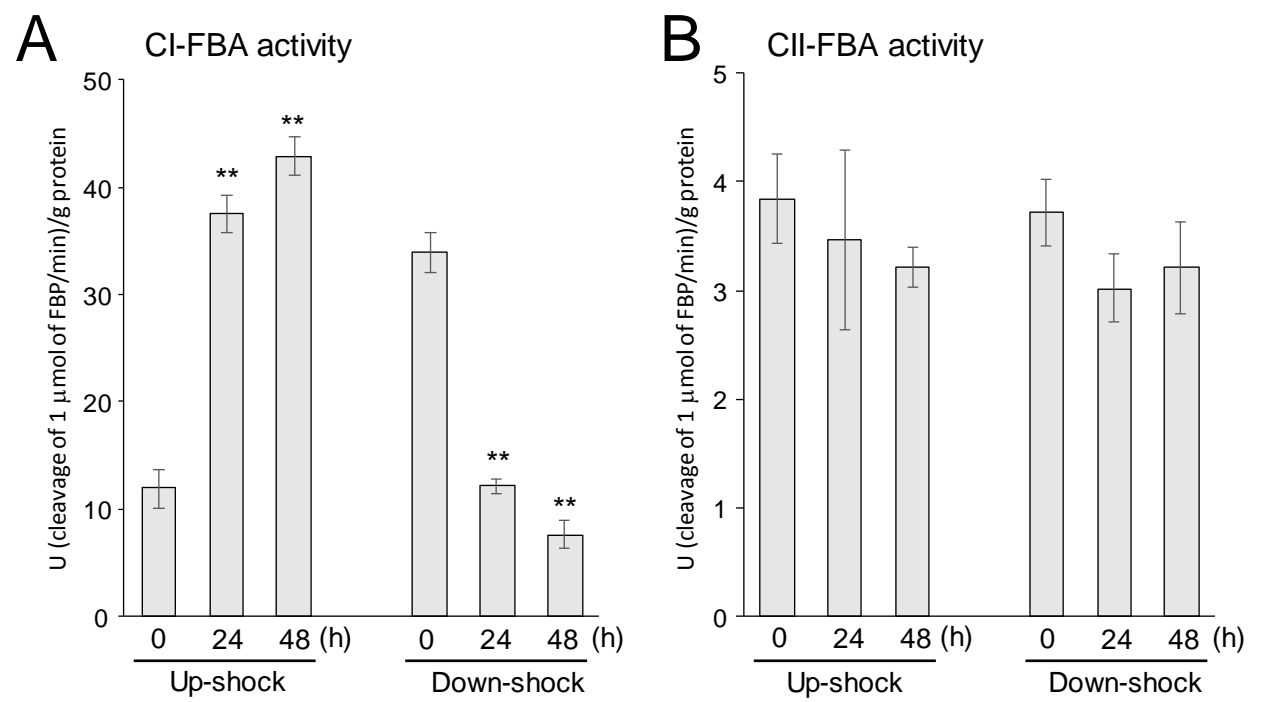

Figure 4. Class I FBA (CI-FBA) (A) and Class II FBA (CII-FBA) (B) activity of the Halothece extracts that were prepared from the cells that received salinity shock treatments. Each value represents an average of three independent analyses. Means \pm SEM are shown. ${ }^{* *}$ indicates that differences between changes in activity compared with time 0 were significant $(t$-test, $p<0.01)$. Note that neither $\mathrm{NaCl}$ nor $\mathrm{KCl}$ was mixed additionally in the reaction mixtures when the activities were measured.

Table 1. Influence of divalent metal ions on FBA activity in the Halothece 7418 extract in the presence of $10 \mathrm{mM}$ sodium borohydride $\left(\mathrm{NaBH}_{4}\right)$.

\begin{tabular}{cccc}
\hline & \multicolumn{2}{c}{ Addition } & \\
\cline { 1 - 3 } Metal Ion $^{2}$ & $\mathbf{N a B H}_{4}{ }^{3}$ & EDTA $^{4}$ & Relative Activity (\%) $^{\mathbf{1}}$ \\
\hline- & - & - & $298 \pm 9.34^{5}$ \\
- & + & - & $\mathrm{ND}^{6}$ \\
$\mathrm{Mg}^{2+}$ & + & - & $73.2 \pm 4.88$ \\
$\mathrm{Ca}^{2+}$ & + & - & $68.3 \pm 5.63$ \\
$\mathrm{Mn}^{2+}$ & + & - & $100 \pm 10.8$ \\
$\mathrm{Fe}^{2+}$ & + & - & $\mathrm{ND}^{6}$ \\
$\mathrm{Co}^{2+}$ & + & - & $\mathrm{ND}^{6}$ \\
$\mathrm{Cu}^{2+}$ & + & - & $46.3 \pm 2.44$ \\
$\mathrm{Zn}^{2+}$ & + & + & $\mathrm{ND}^{6}$ \\
$\mathrm{Mn}^{2+}$ & + & $\mathrm{ND}^{6}$ \\
\hline
\end{tabular}

1 The value obtained when using $\mathrm{Mn}^{2+}$ in the absence of EDTA was set to 100. Data are presented as mean values \pm $\mathrm{SD}$ and were calculated from three sets of independent experiments. ${ }^{2}$ Chloride salts of each indicated metal ion were added to a final concentration of $1 \mathrm{mM} .{ }^{3} \mathrm{NaBH}_{4}$ was added to a final concentration of $10 \mathrm{mM} .{ }^{4}$ EDTA was added to a final concentration of $5 \mathrm{mM} .{ }^{5}$ This activity shows CI-FBA activity in the Halothece extract. ${ }^{6}$ Not detected.

\subsection{NaCl- and KCl-inhibited FBA Activity of Halothece 7418 Extracts}

Halothece 7418 can adapt to the surrounding salt concentration by regulating GB accumulation and unique mechanisms for ion homeostasis in the long term. The intracellular ion content in Halothece 7418 cells that were subjected to salt stress was experimentally determined. Previous reports have revealed low concentrations of intracellular ion contents, although there have been divergences [16]. For instance, Miller et al. reported that the intracellular sodium ion concentration was just $0.027 \mathrm{mM}$ in Halothece 7418 cells that were cultured under $3000 \mathrm{mM} \mathrm{NaCl}$ conditions [17]. Reed et al. demonstrated that sodium and potassium ions were detected at $80-180 \mathrm{mM}$ and $180-250 \mathrm{mM}$, respectively, in Halothece 7418 cells that were cultured under 156-934 mM NaCl conditions [18]. Incharoensakdi and Takabe reported that the intracellular chloride ion concentration in Halothece 7418 ranged from 35 to $150 \mathrm{mM}$ when the $\mathrm{NaCl}$ concentration in the medium was changed from 0.5 to $2.0 \mathrm{M}$ [19]. Thus, the salt concentration of the culture medium can affect the intracellular ion content in Halothece 7418. In addition, when the cells are exposed to a sudden salt shock, the intracellular salt accumulation can be 
transiently changed to achieve a rapid osmotic balance [16]. Intracellular salt might be problematic from an enzymatic point of view; for example, ribulose 1,5-bisphosphate carboxylase-oxygenase (RuBisCO) activity in the crude extracts that were prepared from Halothece 7418 was inhibited by approximately $80 \%$ in the presence of a $300 \mathrm{mM}$ concentration of $\mathrm{NaCl}$ or $\mathrm{KCl}$ [20]. Likewise, the intracellular activities of $\mathrm{H} 2846$ and $\mathrm{H} 2847$ could be affected by these salts. Here, we investigated the effects of $\mathrm{NaCl}$ and $\mathrm{KCl}$ on the activity of FBA in extracts from Halothece 7418 (Figure 5). In these experiments, we tested salt concentrations of up to $300 \mathrm{mM}$. As shown in Figure 5A, NaCl inhibited both CI- and CII-FBA activity in a concentration-dependent fashion. Intriguingly, the resistance of CI-FBA activity to $\mathrm{NaCl}$ was stronger than that of CII-FBA activity. For example, CII-FBA activity was inhibited by $56 \%$, whereas CI-FBA activity was inhibited by just $19 \%$ in the presence of $100 \mathrm{mM} \mathrm{NaCl}$. A similar tendency was observed when using $\mathrm{KCl}$ (Figure 5B). In the presence of $300 \mathrm{mM} \mathrm{KCl}$, CII-FBA activity was reduced to just $12 \%$, but CI-FBA retained $27 \%$ of activity. These observations suggest that CI-FBA H2846 contributes more than CII-FBA H2847 to maintain basic metabolic activities in Halothece 7418 cells under salinity stress conditions. In other words, highly expressed CI-FBA H2846, which has resistance to salts, might compensate for intracellular FBA activity when the constantly expressed housekeeping CII-FBA H2847 is inhibited by salts under salinity stress condition.
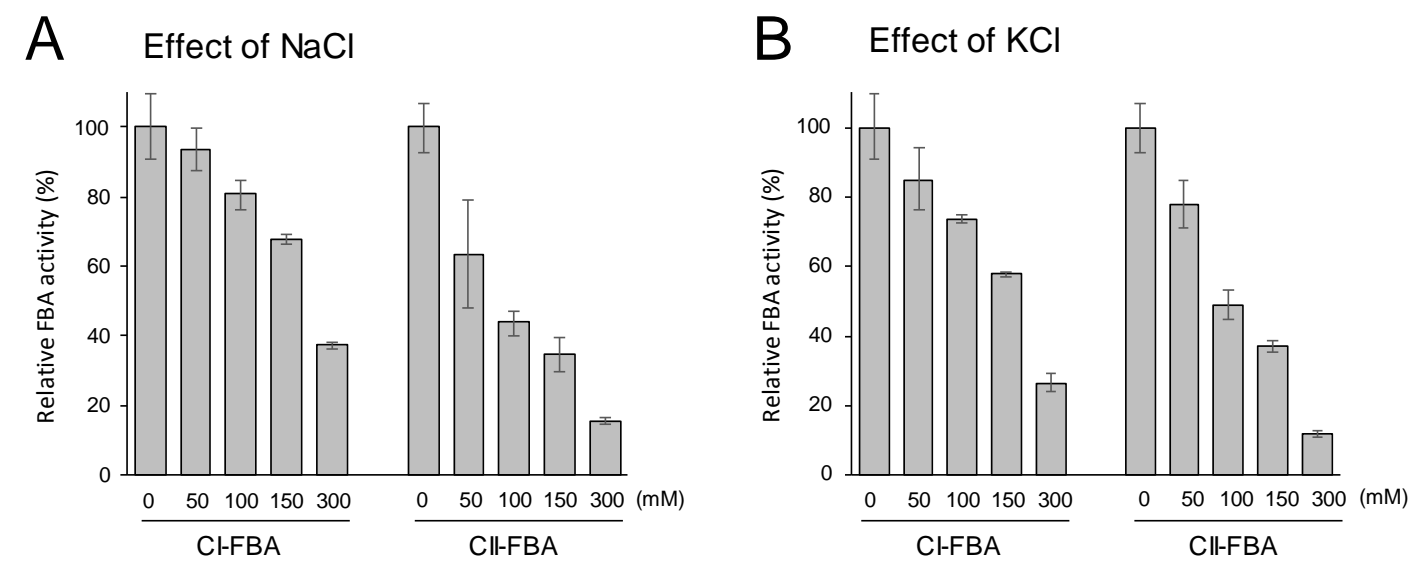

Figure 5. Effects of $\mathrm{NaCl}(\mathbf{A})$ and $\mathrm{KCl}(\mathbf{B})$ on the activity of FBA in the Halothece 7418 extracts. Relative values of CI- and CII-FBA activities are shown. Each value represents an average of three independent analyses. Means \pm SEM are shown. The values at time zero of each dataset were set to 100 .

\subsection{GB Alleviated the Inhibitory Effect of Salts on the CI-FBA Activity of Recombinant H2846 Protein}

Osmoprotectant molecules are important stabilizing agents for macromolecules, including proteins, in cyanobacteria [21]. Halothece 7418 biosynthesizes and accumulates GB in response to salinity stress. In addition to having a strictly osmotic function, GB enhances enzymatic activities in vitro [7] and has been demonstrated to have protective effects against various types of stresses [7,22]. It is worth testing whether GB has any effect on the enzymatic activity of salt-responsive FBAs. We therefore analyzed the effects of GB on CI-FBA activity by using a purified recombinant $\mathrm{H} 2846$ protein. According to previous reports $[18,23]$, the intracellular concentration of GB can be up to $1000 \mathrm{mM}$ in Halothece 7418 . Therefore, we added GB to the reaction mixture at concentrations of 500 and $1000 \mathrm{mM}$. As shown in Figure 6A, the addition of GB alone did not affect the CI-FBA activity of H2846; however, in the presence of $300 \mathrm{mM}$ $\mathrm{NaCl}$ or $\mathrm{KCl}, \mathrm{GB}$ could have reduced the inhibitory effects of both $\mathrm{NaCl}$ and $\mathrm{KCl}$ on CI-FBA activity. In the presence of $\mathrm{KCl}$, in particular, $1000 \mathrm{mM} \mathrm{GB}$ enhanced $\mathrm{KCl}$-inhibited activity by 1.8 times (from 1.2 to $2.2 \mu \mathrm{mol}$ of FBP used/min/mg of protein). Thus, GB might protect $\mathrm{H} 2846$ from potassium ions, since these ions seem to be more abundant than sodium ions in Halothece 7418 cells under high-salinity culture conditions $[17,18,24]$. Though detailed investigation of the protection mechanism of GB awaits further study, the zwitterionic property of GB could contribute to the stabilization of $\mathrm{H} 2846$ by exclusive interaction with its charged groups. Similarly, it has been reported that GB restores the potassium 
ion-induced loss of activity of glucose-6-phosphate dehydrogenase in Halothece 7418 [16]. GB was also shown to protect against $\mathrm{KCl}$-induced damage to RuBisCO activity in Halothece 7418 [23].
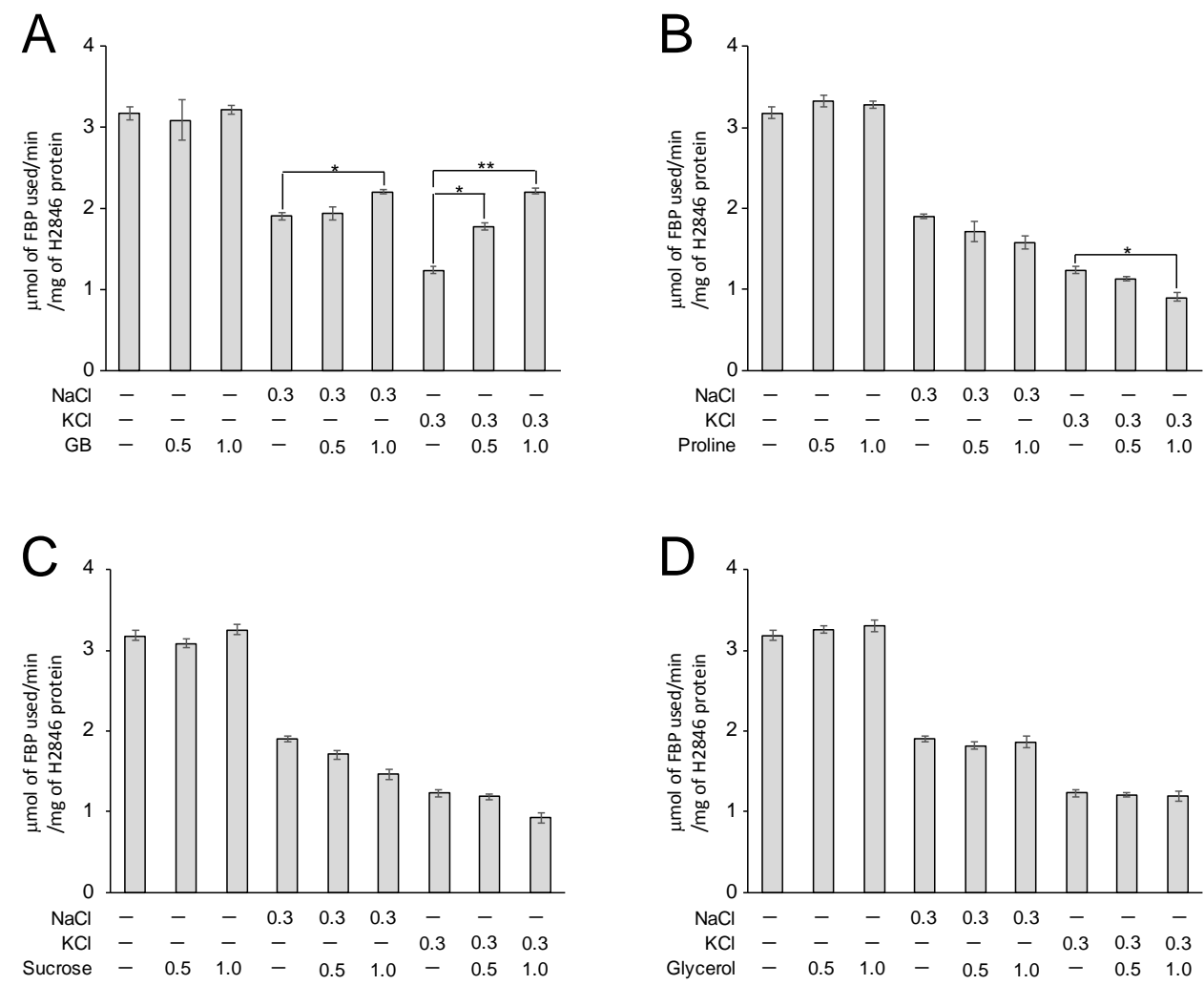

Figure 6. Effects of salts and osmoprotectant molecules on the CI-FBA activity of H2846. A recombinant H2846 protein was used for the activity measurements. The osmoprotectant molecules used were GB (A), proline (B), sucrose (C), and glycerol (D). The final concentration (M) of the added salts and osmoprotectants is indicated. Each value represents the average of three independent analyses. Means \pm SEM are shown. ${ }^{*}$ and ${ }^{* *}$ indicate significant differences between changes in activity $(t$-test, $p<0.05$ and $p<0.01$, respectively).

Given the wide range of chemical properties of osmoprotectants, we employed three potential osmoprotectant molecules (proline, sucrose, and glycerol) to compare their effects with GB. Proline, which is a proteinogenic amino acid, is known to have an osmoprotective function [25]. It was found that the proline content increased in response to salinity concentration in the cyanobacterium Anabaena variabilis [26]. The disaccharide sucrose, as well as trehalose, is known to accumulate as a major compatible solute in freshwater cyanobacterial strains with low halotolerance [27]. Glycerol is well-known as an osmoprotectant that is naturally synthesized in yeast [28]. It has been reported that the cyanobacterium Synechocystis sp. PCC 6803 is also capable of bioproducing glycerol under mild salinity-stress conditions [29]. The most striking observation from using these four osmoprotectants was that only GB showed protective effects for $\mathrm{FBA}$ against $\mathrm{NaCl}$ and $\mathrm{KCl}$ stresses. As shown in Figure 6B-D, the addition of each osmoprotectant alone, at up to $1000 \mathrm{mM}$, did not show any significant effect on the CI-FBA activity of H2846. Likewise, in the case when both osmoprotectant and salt were added, none of the potential osmoprotectant molecules reduced the inhibitory effects of the salts. Note that only in the case where $1000 \mathrm{mM}$ proline was added with $\mathrm{KCl}$ did the CI-FBA activity decrease compared with the condition in the presence of $\mathrm{KCl}$ alone. It is interesting that only GB could protect $\mathrm{H} 2846$ from salts. As described above, the zwitterionic characteristic of GB could affect this phenomenon; however, proline could also exist as zwitterion at physiological $\mathrm{pH}$ values. The structural differences, especially in positively charged parts, between GB and proline might be a reason for different protection effects. 
Thus far, we have not succeeded in preparing recombinant Halothece CII-FBA H2847, as previously mentioned [4]. The enzymatic features of H2847, including effects of GB on its activity, are also interesting; therefore, this area should be examined in the future.

\subsection{Relationship between the Distribution of H2846-type CI-FBA and the Ability to Synthesize GB in Cyanobacteria}

According to the above results, GB could specifically relieve salt damage to CI-FBA H2846, at least among the potential osmoprotectant molecules that were tested. Figure 7 shows a phylogenetic tree of cyanobacterial H2846-like proteins. To depict the tree, we used the top 20 proteins that were hit by the NCBI protein BLAST algorithm, searching with the amino acid sequence of $\mathrm{H} 2846$ as a query. These proteins could be divided into two clades: clades I and II (Figure 7). Halothece 7418 was in clade I, along with cyanobacteria found in hypersaline environments, such as Euhalothece sp. and Dactylococcopsis salina. To explore the relationship between these clades and their ability to synthesize GB, we investigated the distribution of the GB-synthetic enzymes, glycine/sarcosine $N$-methyltransferase (GSMT) and dimethylglycine $N$-methyltransferase (DMT), among the cyanobacteria we analyzed. GSMT and DMT in Halothece 7418 were used as queries for the search. In this investigation, we also noted whether these two methyl transferases were neighboring to each other or not in order to judge the activities of their translation products for GB synthesis because it is known that corresponding genes of GSMT and DMT are adjacent to each other and form clusters in the genome of Halothece 7418, which is one of the best known GB-synthesizing cyanobacterial strains [30]. The results are summarized in Table 2. All species in clade I possessed GSMT- and DMT-like proteins, with similarities of more than $50 \%$ with Halothece GSMT and DMT. This suggested that the strains in clade I synthesized and accumulated GB, except for Cyanobacteria bacterium QS_8_64_29, in which two methyltransferase genes were not neighboring. On the other hand, it seemed that most of the strains in clade II did not possess a complete set of GB synthetic methyltransferases, although confirmation by experiment will be necessary to check that this is indeed the case. It should be noted that GSMT- and DMT-like proteins might be active for GB synthesis in Gloeocapsa sp. PCC 7428 in clade II, because these genes were neighboring in the genome as Halothece 7418, although the homologies were low (Table 2).

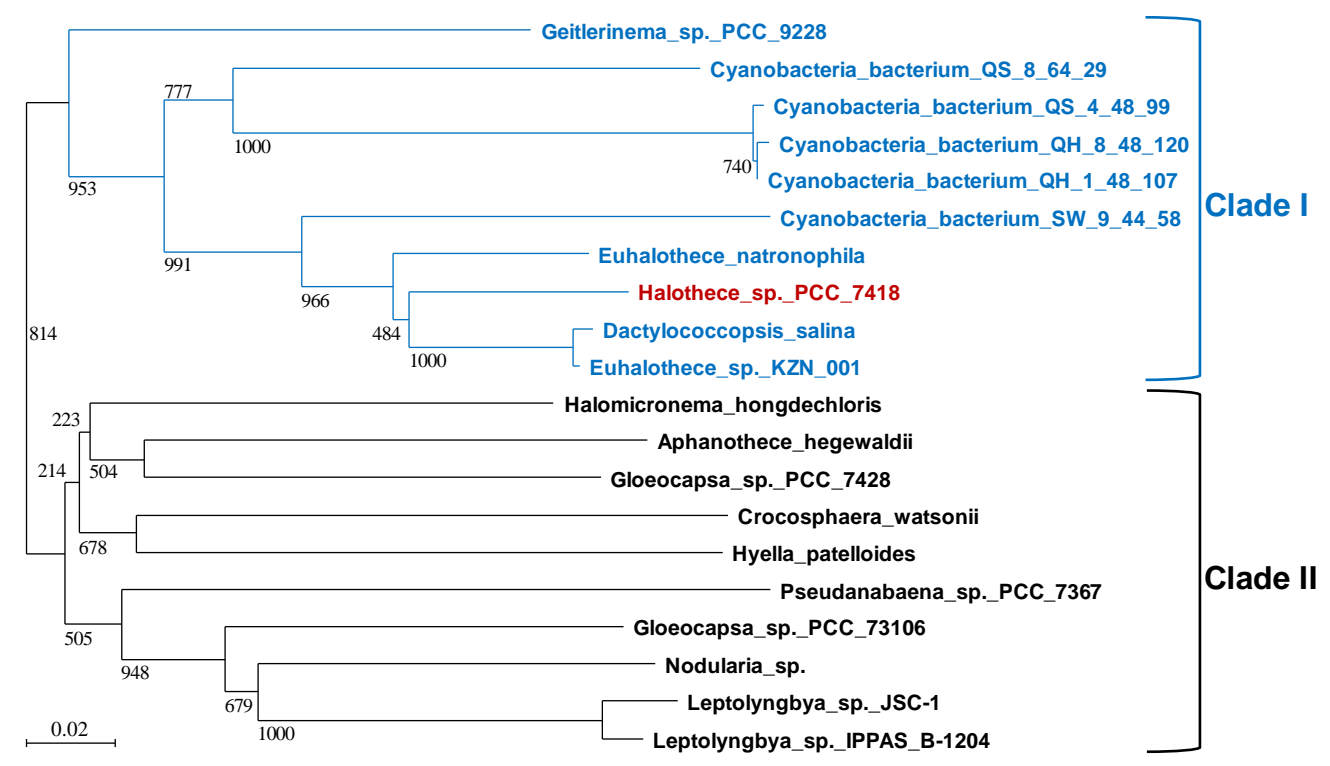

Figure 7. Distribution of the top 20 cyanobacterial strains that possess H2846-type CI-FBA proteins. The tree was generated by the neighbor-joining method by using ClustalW. Strain names in blue indicate cyanobacterial strains in clade I. Note that only Halothece 7418 in clade I is indicated by the color red. Strains in clade II are indicated in black. The bars represent evolutionary distance. The scale bar comprises 0.02 expected changes per amino acid site (0.02 substitutions/site). 
Table 2. Summary of H2846-, glycine/sarcosine N-methyltransferase (GSMT)-, and dimethylglycine N-methyltransferase (DMT)-like proteins in the cyanobacterial strains that were used to generate Figure 7.

\begin{tabular}{|c|c|c|c|c|c|c|c|c|}
\hline \multirow{2}{*}{ Clade } & \multirow{2}{*}{ Cyanobacterial Strain Name } & \multicolumn{2}{|c|}{ H2846-Like Protein } & \multicolumn{2}{|c|}{ GSMT-Like Protein } & \multicolumn{2}{|c|}{ DMT-Like Protein } & \multirow{2}{*}{$\begin{array}{l}\text { Two MT Genes } \\
\text { Are Neighboring }\end{array}$} \\
\hline & & Accession & Identity (\%) & Accession & Identity (\%) & Accession & Identity (\%) & \\
\hline I & Halothece sp. PCC 7418 & WP_015226850.1 & 100.0 & WP_015227493.1 & 100.0 & BAC56940.1 & 100.0 & yes \\
\hline I & Euhalothece sp. KZN 001 & PN̄W51873.1 & 91.35 & PNWW34186.1 & 92.83 & PNW34185.1 & 91.70 & yes \\
\hline I & Dactylococcopsis salina & WP_015230391.1 & 90.78 & WP_015231090.1 & 93.58 & WP_015231089.1 & 91.70 & yes \\
\hline I & Euhalothece natronophila & WP_146295003.1 & 89.91 & WP_146296755.1 & 89.06 & WP_146296756.1 & 94.22 & yes \\
\hline I & Cyanobacteria bacterium SW_9_44_58 & PSO48123.1 & 82.71 & PSO48946.1 & 82.71 & PSO48947.1 & 90.60 & yes \\
\hline I & Cyanobacteria bacterium QS_4_48_99 & PSO81898.1 & 78.10 & PSO78094.1 & 80.84 & PSO78093.1 & 53.09 & yes \\
\hline I & Cyanobacteria bacterium QH_1_48_107 & PSO51072.1 & 78.10 & PSO56755.1 & 80.84 & PSO56754.1 & 53.09 & yes \\
\hline I & Cyanobacteria bacterium QH_8_48_120 & PSO71131.1 & 77.81 & PSO78323.1 & 80.84 & PSO78322.1 & 53.09 & yes \\
\hline I & Geitlerinema sp. PCC 9228 & WP_071515292.1 & 76.68 & WP_071515313.1 & 71.76 & WP_071515314.1 & 57.45 & yes \\
\hline I & Cyanobacteria bacterium QS_8_64_29 & PSP19570.1 & 76.16 & PSP18883.1 & 79.54 & PSP18856.1 & 57.45 & no \\
\hline II & Gloeocapsa sp. PCC 7428 & WP_015191192.1 & 73.70 & WP_015188788.1 & 32.43 & WP_015190787.1 & 50.00 & yes \\
\hline II & Halomicronema hongdechloris & WP_080808545.1 & 73.12 & WP_080807129.1 & 69.88 & WP_080807129.1 & 52.54 & no \\
\hline II & Aphanothece hegewaldii & WP_106456509.1 & 72.25 & N.H. ${ }^{1}$ & - & WP_106456849.1 & 39.39 & no \\
\hline II & Leptolyngbya sp. IPPAS B-1204 & RNJ65497.1 & 71.97 & RNJ64902.1 & 53.12 & RNJ68485.1 & 35.20 & no \\
\hline II & Gloeocapsa sp. PCC 73106 & WP_006527803.1 & 71.80 & WP_006530277.1 & 26.67 & WP_006530803.1 & 31.63 & no \\
\hline II & Pseudanabaena sp. PCC 7367 & WP_015164428.1 & 72.33 & N.H. ${ }^{1}$ & - & WP_015165920.1 & 38.18 & no \\
\hline II & Crocosphaera watsonii & WP_007311889.1 & 71.88 & N.H. ${ }^{1}$ & - & WP_048316702.1 & 36.96 & no \\
\hline II & Leptolyngbya sp. JSC-1 & WP_035999928.1 & 71.39 & WP_051925143.1 & 53.12 & WP_036010558.1 & 35.20 & no \\
\hline II & Nodularia sp. & TVP63635.1 & 70.93 & TVP63914.1 & 29.63 & TVP61242.1 & 36.36 & no \\
\hline II & Hyella patelloides & WP_144874142.1 & 70.52 & WP_144866681.1 & 20.16 & WP_144867980.1 & 33.71 & no \\
\hline
\end{tabular}

${ }^{1}$ N.H., not hi. 


\section{Conclusions}

In conclusion, we found interesting enzymatic properties of the CI-FBA H2846 protein in a halotolerant cyanobacterium, Halothece 7418. It was shown that the accumulation of the $\mathrm{H} 2846$ protein was highly sensitive to both $\mathrm{NaCl}$ up- and down-shock treatments, but it was also found that CII-FBA H2847 remained constant. Corresponding CI-FBA activity in Halothece 7418 extracts also fluctuated sharply in a salinity-dependent manner. In addition, FBA activity in the extracts was significantly inhibited by $\mathrm{NaCl}$ and $\mathrm{KCl}$. Furthermore, the inhibitory effects of salts on the CI-FBA activity of recombinant $\mathrm{H} 2846$ protein were attenuated by the osmoprotectant molecule GB. Finally, it was demonstrated that cyanobacterial CI-FBAs with higher similarities to H2846 tended to be distributed among potential GB-synthesizing cyanobacterial strains. Therefore, H2846-type CI-FBAs are thought to act as metabolism-reinforcing proteins that could be protected by GB, in halotolerant cyanobacteria under high-salinity conditions.

Author Contributions: Conceptualization, R.W.-S. and H.K.; methodology, S.N., M.H., T.P., T.H., R.W.-S. and H.K.; data analysis, S.N., M.H., T.P., T.H., R.W.-S. and H.K.; writing a paper, R.W.-S. and H.K. All authors have read and agreed to the published version of the manuscript.

Funding: This research was funded by Institute for Fermentation, grant number G-2019-3-002.

Acknowledgments: We thank our laboratory members for their helpful discussions.

Conflicts of Interest: The authors declare no conflict of interest.

\section{References}

1. Ziveri, J.; Tros, F.; Guerrera, I.C.; Chhuon, C.; Audry, M.; Dupuis, M.; Barel, M.; Korniotis, S.; Fillatreau, S.; Gales, L.; et al. The metabolic enzyme fructose-1,6-bisphosphate aldolase acts as a transcriptional regulator in pathogenic Francisella. Nat. Commun. 2017, 8, 853. [CrossRef]

2. Berry, A.; Marshall, K.E. Identification of zinc-binding ligands in the Class II fructose-1,6-bisphosphate aldolase of Escherichia coli. FEBS Lett. 1993, 318, 11-16. [CrossRef]

3. Tunio, S.A.; Oldfield, N.J.; Berry, A.; Ala'Aldeen, D.A.A.; Wooldridge, K.G.; Turner, D.P.J. The moonlighting protein fructose-1,6-bisphosphate aldolase of Neisseria meningitidis: Surface localization and role in host cell adhesion. Mol. Microbiol. 2010, 76, 605-615. [CrossRef]

4. Patipong, T.; Ngoennet, S.; Honda, M.; Hibino, T.; Waditee-Sirisattha, R.; Kageyama, H. A class I fructose-1,6-bisphosphate aldolase is associated with salt stress tolerance in a halotolerant cyanobacterium Halothece sp. PCC 7418. Arch. Biochem. Biophys. 2019, 672, 108059. [CrossRef]

5. Ramos, V.M.C.; Castelo-Branco, R.; Leão, P.N.; Martins, J.; Carvalhal-Gomes, S.; Sobrinho da Silva, F.; Mendonça Filho, J.G.; Vasconcelos, V.M. Cyanobacterial diversity in microbial mats from the hypersaline lagoon system of Araruama, Brazil: An in-depth polyphasic study. Front. Microbiol. 2017, 8, 1233. [CrossRef]

6. Waditee-Sirisattha, R.; Kageyama, H.; Sopun, W.; Tanaka, Y.; Takabe, T. Identification and upregulation of biosynthetic genes required for accumulation of mycosporine-2-glycine under salt stress conditions in the halotolerant cyanobacterium Aphanothece halophytica. Appl. Environ. Microbiol. 2014, 80, 1763-1769. [CrossRef]

7. Waditee, R.; Tanaka, Y.; Aoki, K.; Hibino, T.; Jikuya, H.; Takano, J.; Takabe, T.; Takabe, T. Isolation and functional characterization of $\mathrm{N}$-methyltransferases that catalyze betaine synthesis from glycine in a halotolerant photosynthetic organism Aphanothece halophytica. J. Biol. Chem. 2003, 278, 4932-4942. [CrossRef]

8. Patipong, T.; Hibino, T.; Waditee-Sirisattha, R.; Kageyama, H. Efficient bioproduction of mycosporine-2-glycine, which functions as potential osmoprotectant, using Escherichia coli cells. Nat. Prod. Commun. 2017, 12, 1593-1594. [CrossRef]

9. Kageyama, H.; Waditee-Sirisattha, R. Antioxidative, anti-Inflammatory, and anti-aging properties of mycosporine-like amino acids: Molecular and cellular mechanisms in the protection of skin-aging. Mar. Drugs 2019, 17, 222. [CrossRef]

10. Waditee-Sirisattha, R.; Kageyama, H.; Fukaya, M.; Rai, V.; Takabe, T. Nitrate and amino acid availability affects glycine betaine and mycosporine-2-glycine in response to changes of salinity in a halotolerant cyanobacterium Aphanothece halophytica. FEMS Microbiol. Lett. 2015, 362, fnv198. [CrossRef] 
11. Soontharapirakkul, K.; Promden, W.; Yamada, N.; Kageyama, H.; Incharoensakdi, A.; Iwamoto-Kihara, A.; Takabe, T. Halotolerant cyanobacterium Aphanothece halophytica contains an $\mathrm{Na}^{+}$-dependent $\mathrm{F}_{1} \mathrm{~F}_{0}$-ATP synthase with a potential role in salt-stress tolerance. J. Biol. Chem. 2011, 286, 10169-10176. [CrossRef]

12. Kageyama, H.; Waditee-Sirisattha, R.; Sirisattha, S.; Tanaka, Y.; Mahakhant, A.; Takabe, T. Extraction and quantification of alkanes in cyanobacteria. Bio-Protoc. 2015, 5, e1684. [CrossRef]

13. Hobey, W.D.; Prybyla, J.A. Artifacts in Manganese/Tris(hydroxymethyl)aminomethane solutions. Clin. Chem. 1978, 24, 2206-2207. [CrossRef]

14. Siebers, B.; Brinkmann, H.; Dörr, C.; Tjaden, B.; Lilie, H.; van der Oost, J.; Verhees, C.H. Archaeal fructose-1,6-bisphosphate aldolases constitute a new family of archaeal type class I aldolase. J. Biol. Chem. 2001, 276, 28710-28718. [CrossRef]

15. Nakahara, K.; Yamamoto, H.; Miyake, C.; Yokota, A. Purification and characterization of class-I and class-II fructose-1,6-bisphosphate aldolases from the cyanobacterium Synechocystis sp. PCC6803. Plant Cell Physiol. 2003, 44, 326-333. [CrossRef]

16. Oren, A. Salt and brines. In The Ecology of Cyanobacteria, 1st ed.; Whitton, B.A., Potts, M., Eds.; Springer: Dordrecht, The Netherlands, 2000; pp. 281-306.

17. Miller, D.M.; Jones, J.H.; Yopp, J.H.; Tindall, D.R.; Schmid, W.E. Ion metabolism in a halophilic blue-green alga, Aphanothece halophytica. Arch. Microbiol. 1976, 111, 145-149. [CrossRef]

18. Reed, R.H.; Chudek, J.A.; Foster, R.; Stewart, W.D.P. Osmotic adjustment in cyanobacteria from hypersaline environments. Arch. Microbiol. 1984, 138, 333-337. [CrossRef]

19. Incharoensakdi, A.; Takabe, T. Determination of intracellular chloride ion concentration in a halotolerant cyanobacterium Aphanothece halophytica. Plant Cell Physiol. 1988, 29, 1073-1075.

20. Takabe, T.; Rai, A.K.; Akazawa, T. Interaction of constituent subunits in Ribulose 1,5-bisphosphate carboxylase from Aphanothece halophytica. Arch. Biochem. Biophys. 1984, 229, 202-211. [CrossRef]

21. Klähn, S.; Hagemann, M. Compatible solute biosynthesis in cyanobacteria. Environ. Microbiol. 2011, 13, 551-562. [CrossRef]

22. Zou, H.; Wu, Z.; Xian, M.; Liu, H.; Cheng, T.; Cao, Y. Not only osmoprotectant: Betaine increased lactate dehydrogenase activity and L-lactate production in lactobacilli. Bioresour. Technol. 2013, 148, 591-595. [CrossRef]

23. Incharoensakdi, A.; Takabe, T.; Akazawa, T. Effect of betaine on enzyme activity and subunit interaction of riblose-1,5-bisphosphate carboxylase/oxygenase from Aphanothece halophytica. Plant Physiol. 1986, 81, 1044-1049. [CrossRef]

24. Oren, A. Salts and brines. In Ecology of Cyanobacteria II: Their diversity in Space and Time, 1st ed.; Whitton, B.A., Ed.; Springer: Heidelberg, Germany, 2012; pp. 401-425.

25. Szabados, L.; Savouré, A. Proline: A multifunctional amino acid. Trends Plant Sci. 2009, 15, 89-97. [CrossRef]

26. Syiem, M.B.; Nongrum, N.A. Increase in intracellular proline content in Anabaena variabilis during stress conditions. J. Appl. Nat. Sci. 2011, 3, 119-123. [CrossRef]

27. Kolman, M.A.; Nishi, C.N.; Perez-Cenci, M.; Salerno, G.L. Sucrose in cyanobacteria: From a salt-response molecule to play a key role in nitrogen fixation. Life 2015, 5, 102-126. [CrossRef]

28. Lai, M.C.; Lan, E.I. Advances in metabolic engineering of cyanobacteria for photosynthetic biochemical production. Metabolites 2015, 5, 636-658. [CrossRef]

29. Savakis, P.; Tan, X.; Du, W.; Branco dos Santos, F.; Lu, X.; Hellingwerf, K.J. Photosynthetic production of glycerol by a recombinant cyanobacterium. J. Biotechnol. 2015, 195, 46-51. [CrossRef]

30. Waditee-Sirisattha, R.; Singh, M.; Kageyama, H.; Sittipol, D.; Rai, A.K.; Takabe, T. Anabaena sp. PCC7120 transformed with glycine methylation genes from Aphanothece halophytica synthesized glycine betaine showing increased tolerance to salt. Arch. Microbiol. 2012, 194, 909-914. [CrossRef]

(C) 2020 by the authors. Licensee MDPI, Basel, Switzerland. This article is an open access article distributed under the terms and conditions of the Creative Commons Attribution (CC BY) license (http://creativecommons.org/licenses/by/4.0/). 